\title{
APLICATIVOS PARA TREINO COGNITIVO: UMA REVISÃO SISTEMÁTICA
}

\author{
Emanoel Fontana - Ciência da Computação/UPF - 138031@ upf.br \\ Ana Carolina B. De Marchi - PPGCA/UPF - carolina@ upf.br
}

\begin{abstract}
Resumo - O treino cognitivo é uma prática que pode minimizar dificuldades de aprendizagem, de memorização e de atenção. Dentre as estratégias adotadas nos treinos encontra-se o uso de tecnologias, as quais potencializam a motivação dos participantes, aumentando a adesão. O objetivo deste estudo foi identificar e investigar aplicativos móveis para treino cognitivo. Trata-se de uma revisão sistemática da literatura realizada nas bases de dados IEEE Xplore, ScienceDirect, Springer Link, CiteSeerX, ACM DL e Google Scholar, de 04 a 11 de maio de 2016. Dos 277 estudos identificados, apenas oito atenderam aos critérios de elegibilidade. Após análise dos trabalhos foi possível perceber a importância do desenvolvimento de aplicações móveis para o treino cognitivo, pois estimulam os usuários a exercer o treino diariamente.
\end{abstract}

Palavras-chaves - aplicações móveis; treino cognitivo; transtornos de memória; transtornos de atenção; informática em saúde;

\section{Applications for cognitive training: a systematic review}

\begin{abstract}
Cognitive training is a practice that can minimize learning, memorization and attention difficulties. Among the strategies adopted in the trainings are the use of technologies, which potentiate the motivation of the participants, increasing the adhesion. The aim of this study was to identify and investigate mobile applications for cognitive training. This is a systematic review of the literature carried out in the IEEE Xplore, ScienceDirect, Springer Link, CiteSeerX, ACM DL and Google Scholar databases, from 04 the 11 May 2016. Of the 277 studies identified, only eight met eligibility criteria. After analyzing the work, it was possible to perceive the importance of the development of mobile applications for the cognitive training, since they stimulate the users to exercise the training daily.
\end{abstract}

Keywords - mobile applications; cognitive training; memory disorders; attention disorders; health informatic;

\section{Introdução}

A cognição é um processo complexo que envolve, entre outros, a percepção, a atenção, a memória e a linguagem. Muitos são os fatores que afetam o sistema cognitivo, os quais podem se manifestar desde a infância até o envelhecimento. Dentre eles destacam-se o Transtorno de Déficit de Atenção e Hiperatividade (TDAH), presente em algumas crianças e responsável por um conjunto de prejuízos médico-sociais especialmente dificuldades escolares (Possa et al., 2005); e o declínio do processamento e da memória, reflexo normal do envelhecimento humano que resulta em dificuldades no desempenho de atividades comuns da vida diária (Assis et al., 2015).

Estudos demostram que é possível mobilizar, ou até mesmo melhorar, os déficits ocasionados pelo envelhecimento ou por TDAH por meio de treino cognitivo (Buiza et 
al., 2009; Bless et al., 2014), tendo como foco o treinamento de funções cognitivas (Irigaray et al., 2012).

Atualmente, muitas pesquisas vêm utilizando tecnologias para o treino cognitivo, como videogames (Torres, 2008; Assis et al., 2015; Martel et al., 2016) e aplicativos móveis (Bless et al., 2014; Caron et al., 2015). Segundo Aranha (2006), muitos destes softwares são desenvolvidos na forma de jogos para ampliar o interesse de seus usuários. Os aplicativos para treino cognitivo tentam seguir os princípios de usabilidade como: tempo de aprendizagem, desempenho, facilidade de memorização e satisfação do usuário. Quando levados em consideração, estes princípios evitam certos problemas para o usuário, tais como: treinamento excessivo para aprendizado, insatisfação do usuário e diminuição de produtividade (Silva, 2015), aumentando, com isso, a motivação no uso.

Diante deste contexto, este trabalho teve como propósito revisar sistematicamente a literatura de modo a identificar aplicativos móveis utilizados para treino cognitivo. Com o objetivo de analisar os estudos primários encontrados, a revisão buscou demonstrar especificações e modelos experimentais dos aplicativos, além de verificar os princípios de usabilidade empregados.

Este artigo está organizado em quatro seções: a Seção 2 apresenta a metodologia da revisão sistemática da literatura e as etapas de desenvolvimento do estudo, a Seção 3 apresenta os resultados obtidos, enquanto na Seção 4 os discute. Por fim, a Seção 5 apresenta a conclusão e os trabalhos futuros.

\section{Metodologia}

Trata-se de uma Revisão Sistemática da Literatura (RSL), que, de acordo com Castro (2001), é planejada para responder a uma pergunta específica. Ainda de acordo com o autor, o processo utiliza métodos explícitos e sistemáticos para identificar, selecionar e avaliar criticamente estudos primários, e para coletar e analisar os dados dos estudos incluídos na revisão.

Neste sentido, a questão de pesquisa que norteou este trabalho foi: quais os aplicativos existentes para treino cognitivo? Para responder a questão foram elencados alguns termos em língua inglesa que permitiram a definição da seguinte estratégia de busca: ("training" OR "practice" OR "exercises") AND ("cognitive" OR "learning" OR "knowledge") AND ("mobile applications" OR "electronic games" OR "tablets" OR "smartphones").

As buscas foram realizadas em seis bases de dados, a saber: ScienceDirect, ACM Digital Library, IEEE Xplore Digital Library, Springer Link, Biblioteca digital de pesquisa científica CiteSeerX e referências acadêmicas presentes no Google Scholar.

Para a inclusão do artigo na análise final, o mesmo deveria atender a pelo menos um dos critérios de elegibilidade abaixo:

- descrever o desenvolvimento do aplicativo utilizado para treino cognitivo;

- testar a usabilidade do aplicativo de treino cognitivo;

- avaliar o uso de tecnologias para treino cognitivo;

Efetuada a busca nas bases citadas, obteve-se como retorno 277 trabalhos, sendo 163 oriundos da base de pesquisa Google Scholar, 35 da ScienceDirect, 6 da ACM DL Digital Library, 3 da IEEE Xplore Digital Library, 52 da base de pesquisa Springer Link e 18 da Biblioteca digital de pesquisa científica CiteSeerX. Esta primeira fase de busca dos estudos foi realizada entre os dias 04 e 11 de maio de 2016. Foram descartados os 
artigos duplicados.

A partir de então foi realizada uma primeira triagem dos estudos que se sucedeu a partir da análise dos títulos das obras, em função dos critérios de elegibilidade. Tomou-se como filtro a existência de, pelo menos, um termo presente no título com relação ao âmbito de treino cognitivo associado ao uso de tecnologias. Esta atividade culminou num montante de 32 artigos.

A segunda triagem se deu com a leitura dos resumos dos artigos, onde foram excluídos nove trabalhos, os quais não atendiam aos critérios de elegibilidade. Permaneceram 21 trabalhos para uma análise na íntegra.

$\mathrm{Na}$ terceira triagem foi efetuada a leitura completa dos materiais, totalizando na inclusão final de oito trabalhos. Portanto, este artigo baseia-se nas informações oriundas destes oito trabalhos restantes do processo de RSL. O processo descrito pode ser visualizado na Tabela 1.

Tabela 1 - Tabela de resultados da revisão sistemática

\begin{tabular}{|l|c|c|c|c|}
\hline Bases de pesquisa & $\begin{array}{c}\text { Resultados } \\
\text { totais }\end{array}$ & $\begin{array}{c}\text { Selecionados 1 } \\
\text { triagem }\end{array}$ & $\begin{array}{c}\text { Selecionados 2 } \\
\text { triagem }\end{array}$ & $\begin{array}{c}\text { Selecionados } \mathbf{3}^{\mathbf{a}} \\
\text { triagem }\end{array}$ \\
\hline Google Scholar & 163 & 5 & 3 & 1 \\
\hline ScienceDirect & 35 & 12 & 8 & 3 \\
\hline ACM Digital Library & 6 & 2 & 1 & 0 \\
\hline IEEE Xplore Digital & 3 & 3 & 2 & 2 \\
\hline Library & 52 & 7 & 5 & 2 \\
\hline Springer Link & 18 & 3 & 2 & 0 \\
\hline CiteSeerX & $\mathbf{2 7 7}$ & $\mathbf{3 2}$ & $\mathbf{2 1}$ & $\mathbf{8}$ \\
\hline TOTAL & & & & \\
\hline
\end{tabular}

A Tabela 2 apresenta os artigos que foram selecionados após a terceira triagem, os quais foram analisados e organizados pelos autores, de acordo com os critérios de elegibilidade.

Tabela 2 - Artigos selecionados e análise dos critérios de inclusão

\begin{tabular}{|c|c|c|c|}
\hline Estudo & $\begin{array}{c}\text { Descrição do } \\
\text { desenvolvimento de } \\
\text { aplicativos utilizados } \\
\text { para treino cognitivo }\end{array}$ & $\begin{array}{c}\text { Teste de usabilidade de } \\
\text { aplicativos de treino } \\
\text { cognitivo }\end{array}$ & $\begin{array}{c}\text { Avaliação do uso de } \\
\text { tecnologias para treino } \\
\text { cognitivo }\end{array}$ \\
\hline Ogomori et al. (2011) & $\checkmark$ & & $\checkmark$ \\
\hline Clarebout et al. (2015) & & $\checkmark$ & $\checkmark$ \\
\hline Pascual et al. (2012) & $\checkmark$ & $\checkmark$ & $\checkmark$ \\
\hline Buiza et al. (2009) & & & \\
\hline Wittland et al. (2015) & $\checkmark$ & & $\checkmark$ \\
\hline Agheana, V. e Duță, N. & $\checkmark$ & & \\
\hline Yue et al. (2015) & $\checkmark$ & & \\
\hline Bless et al. (2014) & $\checkmark$ & & \\
\hline
\end{tabular}




\section{Resultados}

Os oito estudos eleitos não apresentaram uma padronização quanto às técnicas utilizadas nas aplicações. Diante disso, não foi possível realizar uma análise estatística dos resultados das avaliações de usabilidade.

Cada estudo será apresentado buscando atender aos seguintes itens: a) objetivo do trabalho; b) tecnologias utilizadas; c) de que forma, como ou para que é empregado o treino cognitivo; e e) resultados dos trabalhos e o que os autores concluíram. A Tabela 3 apresenta um panorama resumido dos estudos analisados.

Tabela 3 - Comparativo dos resultados da revisão sistemática

\begin{tabular}{|c|c|c|c|c|}
\hline Estudo & Objetivo do trabalho & $\begin{array}{l}\text { Tecnologias } \\
\text { usadas }\end{array}$ & $\begin{array}{c}\text { Como e para que } \\
\text { foi usada a } \\
\text { tecnologia } \\
\end{array}$ & Resultados e conclusões \\
\hline $\begin{array}{l}\text { Ogomori et } \\
\text { al. }(2011)\end{array}$ & $\begin{array}{l}\text { Demonstrar os } \\
\text { requisitos de um jogo } \\
\text { para treino cognitivo }\end{array}$ & $\begin{array}{l}\text { Computadores, } \\
\text { Smartphones e } \\
\text { aparelhos } \\
\text { eletromecânicos } \\
\end{array}$ & $\begin{array}{l}\text { Treino de } \\
\text { memória para } \\
\text { idosos }\end{array}$ & $\begin{array}{c}\text { O estímulo do jogador é } \\
\text { importante para que o treino } \\
\text { de memória (jogo) tenha mais } \\
\text { eficiência }\end{array}$ \\
\hline $\begin{array}{l}\text { Clarebout et } \\
\text { al. (2015) }\end{array}$ & $\begin{array}{l}\text { Demonstrar o uso de } \\
\text { tecnologia na educação } \\
\text { com bases cognitivas }\end{array}$ & iPods & $\begin{array}{l}\text { Aprendizagem de } \\
\text { crianças em idade } \\
\text { escolar }\end{array}$ & $\begin{array}{l}\text { O uso da tecnologia em } \\
\text { paralelo a outra atividade para } \\
\text { ensino merce atenção em } \\
\text { alunos com TDAH }\end{array}$ \\
\hline $\begin{array}{l}\text { Pascual et } \\
\text { al. }(2012)\end{array}$ & $\begin{array}{l}\text { Diagnosticar e tratar } \\
\text { pacientes com TDAH }\end{array}$ & $\begin{array}{l}\text { Smartphones, } \\
\text { Linguagem Java }\end{array}$ & $\begin{array}{c}\text { Testar se } \\
\text { adolescentes } \\
\text { sofrem de TDAH }\end{array}$ & $\begin{array}{c}\text { Há um maior interesse por } \\
\text { parte dos usuários na } \\
\text { utilização de testes que } \\
\text { envolvem tecnologia }\end{array}$ \\
\hline $\begin{array}{l}\text { Buiza et al. } \\
\text { (2009) }\end{array}$ & $\begin{array}{c}\text { Avaliar as vantagens do } \\
\text { uso de tecnologias para } \\
\text { o treino cognitivo }\end{array}$ & $\begin{array}{l}\text { Tablets, } \\
\text { Smartphones }\end{array}$ & $\begin{array}{l}\text { Treino de } \\
\text { memória em } \\
\text { idosos } \\
\end{array}$ & $\begin{array}{c}\text { O uso da tecnologia é } \\
\text { eficiente para o treino } \\
\text { cognitivo }\end{array}$ \\
\hline $\begin{array}{l}\text { Wittland et } \\
\text { al. }(2015)\end{array}$ & $\begin{array}{l}\text { Demonstrar o } \\
\text { desenvolvimento de um } \\
\text { serious games para } \\
\text { treino cognitivo }\end{array}$ & Tablets, Kinect & $\begin{array}{l}\text { Treino de } \\
\text { memória em } \\
\text { idosos }\end{array}$ & $\begin{array}{l}\text { Os jogos sérios são muito } \\
\text { eficazes para o treino } \\
\text { cognitivo, por serem mais } \\
\text { motivadores e atraentes aos } \\
\text { usuários }\end{array}$ \\
\hline $\begin{array}{l}\text { Agheana, } \\
\text { V.. Duță, N. } \\
\text { (2015) }\end{array}$ & $\begin{array}{l}\text { Mostrar a utilização de } \\
\text { tablets para } \\
\text { aprendizagem de } \\
\text { portadores de síndrome } \\
\text { de Down } \\
\end{array}$ & Tablets & $\begin{array}{l}\text { Aprendizagem de } \\
\text { crianças } \\
\text { portadoras de } \\
\text { síndrome de } \\
\text { Down } \\
\end{array}$ & $\begin{array}{c}\text { O uso de tecnologia na } \\
\text { educação é uma maneira de } \\
\text { melhorar e estimular a forma } \\
\text { de aprendizagem }\end{array}$ \\
\hline $\begin{array}{l}\text { Yue et al. } \\
\text { (2013) }\end{array}$ & $\begin{array}{l}\text { Ensinar e treinar a } \\
\text { linguagem falada com } \\
\text { jogos móveis }\end{array}$ & $\begin{array}{c}\text { Jogos, comandos } \\
\text { de voz, tecnologia } \\
\text { móvel }\end{array}$ & $\begin{array}{l}\text { Ensino e treino da } \\
\text { linguagem falada } \\
\text { para crianças }\end{array}$ & $\begin{array}{c}\text { O uso de jogos para } \\
\text { aprendizagem da linguagem } \\
\text { falada é uma maneira } \\
\text { interessante para um treino } \\
\text { constante, porém são } \\
\text { necessários mais estudos } \\
\text { sobre o assunto }\end{array}$ \\
\hline $\begin{array}{l}\text { Bless et al. } \\
\quad(2014)\end{array}$ & $\begin{array}{l}\text { Treinar a atenção com } \\
\text { aplicativos móveis }\end{array}$ & Tecnologia móvel & $\begin{array}{l}\text { Treino de atenção } \\
\text { e audição em } \\
\text { pessoas idosas }\end{array}$ & $\begin{array}{l}\text { O estudo verificou a atenção } \\
\text { dos usuários através de efeitos } \\
\text { sonoros, porém notou-se que } \\
\text { para idosos há dificuldades de } \\
\text { usabilidade, por eles não se } \\
\text { adaptarem a utilização de } \\
\text { fones }\end{array}$ \\
\hline
\end{tabular}


O trabalho de Ogomori et al. (2011) lista requisitos necessários de um jogo para o treino cognitivo, além de descrever o desenvolvimento do jogo de memória Ikiikipom, destinado a idosos e/ou pessoas com deficiência cognitiva. O jogo tem botões com cores diferentes e o objetivo é repetir a sequência aleatória de luzes acessas a cada jogada. O estudo leva em consideração a motivação do jogador, apostando que um jogo estimulante faz com que o usuário queira jogar repedidas vezes, e, com isso, aumente a concentração e treine a memória.

Para a utilização de tecnologia na educação é importante levar em conta os aspectos cognitivos, que se preocupam em compreender como os alunos processam diferentes tarefas, como prestam atenção, e, consequentemente, o efeito na aprendizagem. Desta maneira, Clarebout et al. (2015) apresentam perspectivas cognitivas a partir do uso de iPods na educação e considerando pontos positivos e negativos na aprendizagem móvel. No trabalho foram realizados testes para verificar se o uso desta tecnologia interfere ou não no processo de estudo. Os iPods foram utilizados para testar a atenção dos alunos. Um grupo de 23 alunos utilizou apenas o iPod para realização de uma atividade escolar, enquanto outro grupo de 27 alunos utilizou o iPod e realizou outra tarefa simultaneamente. Os resultados demonstraram que os alunos com TDAH não apresentaram o mesmo desempenho que os demais, sendo necessário mais tempo de treinamento. $O$ estudo não especificou o aplicativo utilizado, apenas apresentou os testes obtidos a partir do uso da tecnologia.

Com o objetivo de diagnosticar e tratar crianças e adolescentes com TDAH, Pascual et al. (2012) propuseram um jogo eletrônico com base em testes psicológicos tradicionais. Os autores desenvolveram, na linguagem Java, uma adaptação tecnológica do teste D2, um teste psicológico que tradicionalmente utiliza lápis e papel. Assim como no teste tradicional, a adaptação mede dois tipos diferentes de falta de atenção: atenção seletiva, que é a capacidade que o usuário tem em manter o foco em um objeto específico; e atenção concentrada, que é a capacidade que o usuário tem em permanecer focado durante um período de tempo. Dessa maneira, para avaliar a eficácia do projeto foram realizados testes de usabilidade em um grupo de usuários com idades entre $12 \mathrm{e}$ 18 anos. Os resultados da avaliação foram obtidos através da observação de terapeutas, e também com o uso de questionários para verificar a satisfação do usuário, sendo este baseado na usabilidade do sistema.

No estudo de Buiza et al. (2009) foi avaliado o uso de tecnologias para o treino cognitivo, verificando se é ou não benéfico aos usuários. O estudo avaliou jogos de treino de memória tradicionais e jogos digitais: jogos de labirinto e quebra-cabeça. Ambos apresentaram a mesma eficiência ao estímulo da memória, todavia, os autores perceberam que os jogos digitais incentivam os usuários a jogá-los diariamente, possibilitando melhoras maiores que os jogos tradicionais. Foram realizados testes de usabilidade, onde um grupo de usuários-alvo utilizou os aplicativos e os avaliou por meio de entrevistas individuais. Desta forma, foi possível mostrar prioridades, interesses, motivações ou ansiedades dos usuários, os dados obtidos nos testes usabilidade não foram especificados no trabalho.

Jogos que tem uma finalidade específica para uma determinada área do conhecimento que vão além do entretenimento, são chamados de serious game (jogos sérios). Existe uma forte iniciativa para se aplicar o uso de jogos sérios no cotidiano das pessoas permitindo o treinamento domiciliar, além de permitir ser utilizados para 
diferentes tipos de treino cognitivo e com qualquer tipo de tecnologia (Wittland et al., 2015).

Wittland et al. (2015) utilizaram um serious game para treino cognitivo em ambientes de vida assistida. O trabalho apresentou o jogo Cook It Right, desenvolvido a partir de storyboards e protótipos de papel para possibilitar a captura de ideias geradas pelos usuários e transformá-las em um jogo adequado. $\mathrm{O}$ jogo tem como objetivo simular o preparo de uma refeição. Para tanto, é fornecida uma receita e o usuário precisa procurar os ingredientes da mesma. De acordo com os autores, o jogo teve resultados semelhantes a jogos de treino cognitivo tradicionais, porém essa avaliação reflete dados de curto prazo de interação do usuário com o jogo, sendo necessária uma avaliação a longo prazo. Durante o desenvolvimento do projeto foram levados em consideração os critérios de usabilidade para suprir as necessidades dos idosos, porém não foram realizados testes de usabilidade do produto final.

Crianças com síndrome de Down têm dificuldades no desenvolvimento de atividades cognitivas. Para poder melhor a capacidade de aprendizagem destas crianças, o estudo de Agheana e Duță (2015) teve como objetivo ensinar matemática a crianças com síndrome de Down. Para tanto, foram desenvolvidos aplicativos matemáticos sobre contagem e aritmética básica. Estes aplicativos, instalados em tablets, foram aplicados a um grupo de doze crianças com síndrome de Down. Os resultados apontaram um maior estímulo e maior aprendizagem com a utilização do aplicativo.

No estudo de Yue et al. (2013) foi proposto o desenvolvimento de um aplicativo móvel para o ensino de crianças que sofrem de problemas auditivos. O aplicativo móvel desenvolvido em Android, nomeado Handicaps, tem como objetivo ensinar e treinar a linguagem falada a crianças com dificuldades auditivas. Ademais, o aplicativo tem percursos que são necessários comandos de voz, fazendo com que o usuário tente falar corretamente. $\mathrm{O}$ estudo obteve algumas estratégias de teorias de mudança de comportamento comprovada como: interesse crescente da aprendizagem, motivação e atitude positiva dos usuários em estudar. $\mathrm{O}$ trabalho propôs realizar testes de usabilidade para combinar as funcionalidades do aplicativo com as necessidades dos usuários.

Por fim, o trabalho de Bless et al. (2014) apresentou um aplicativo, em desenvolvimento, de treino cognitivo e de atenção auditiva. $O$ aplicativo, com a utilização de fones de ouvido, explora a atenção auditiva do usuário. Em um lado do fone é falada a sílaba que o usuário deve clicar, enquanto o outro lado do fone emite outras sílabas. Antes das sílabas serem ouvidas é avisado ao usuário o lado do fone que eles devem prestar atenção, para posteriormente clicar na sílaba falada por ele. Segundo os autores, a capacidade de se concentrar na entrada auditiva durante condições de estímulo concorrentes foi aumentada.

\section{Discussão}

Os trabalhos analisados tiveram como objetivo o uso de tecnologia para o treino cognitivo. Os estudos demonstraram comparativos entre métodos tradicionais de treino cognitivo e treinos por meio de jogos. Também, apresentaram diferentes formar de utilizar a tecnologia em treino cognitivo, podendo ser usado para treino de memória, treino de atenção e aprendizagem. Dos estudos analisados $37,5 \%$ focaram no treino de memória, $37,5 \%$ no uso para a aprendizagem, e $25 \%$ para o treino de atenção, conforme ilustra a Figura 1. 


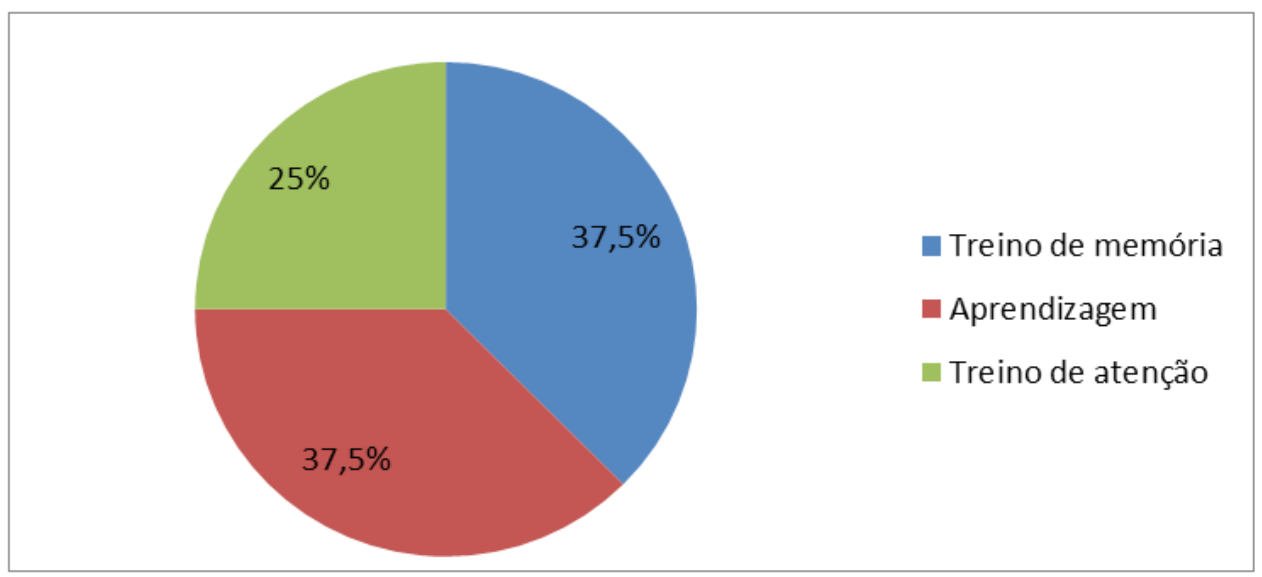

Figura 1. Áreas de aplicação do uso das tecnologias para treino cognitivo.

De todos os trabalhos, apenas dois (25\%) não apresentaram o desenvolvimento de uma aplicação móvel. Os trabalhos fizeram uma avaliação do uso de tecnologias para treino cognitivo, Buiza (2009) também realizou testes de usabilidade para garantir as necessidades de seu público-alvo.

Os seis trabalhos que apresentaram aplicativos para treino cognitivo (75\% dos casos) relataram sucesso em seus resultados, especialmente quanto à motivação e ao engajamento dos usuários.

No decorrer do estudo, observou-se que o uso de tecnologias para o treino cognitivo pode ser categorizado em áreas distintas, como para o envelhecimento humano, e para o transtorno de déficit de atenção. Dos trabalhos analisados, quatro estudaram tecnologias no campo do envelhecimento humano, três na área de TDAH, e um voltado ao desenvolvimento de crianças portadoras de síndrome de Down.

Outro fator que pode ser considerado importante é que os estudos se preocuparam com questões de usabilidade e experiência do usuário. Durante o processo de desenvolvimento, alguns trabalhos já consideravam as necessidades dos usuários.

Entretanto, pode-se dizer que tais pesquisas ainda estão em seu estágio inicial de desenvolvimento, uma vez que as interfaces propostas podem ser melhoradas, tanto em qualidade visual, como no uso de dispositivos e técnicas que estimulem ainda mais a interação com o sistema por parte do usuário.

\section{Conclusão}

Esse trabalho buscou apresentar uma revisão sistemática que contempla trabalhos relacionados sobre a questão de pesquisa: quais os aplicativos existentes para treino cognitivo?

Ao longo do estudo, percebeu-se a importância do desenvolvimento de aplicações móveis para o treino cognitivo. Estas estimulam os usuários a exercer o treino diariamente, o que beneficia no processo de envelhecimento, por minimizar o declínio da memória e, o TDAH, pois melhora a capacidade de concentração.

Com a finalidade de aprofundar e dar continuidade as temáticas do artigo, será desenvolvido um aplicativo para treino de atenção. O aplicativo levará em considerações os dados estudados e analisados nos trabalhos encontrados nesta RSL.

\section{Agradecimentos}

Ao Conselho Nacional de Desenvolvimento Científico e Tecnológico - CNPq. 


\section{Referências}

AGHEANA, V.; DUȚ $\breve{A}$, N.. "Achievements of Numeracy Abilities to Children with Down Syndrome: Psycho-Pedagogical Implications", Procedia - Social and Behavioral Sciences, v. 186, p. 38-45, 2015.

ARANHA, G. "Jogos Eletrônicos como um conceito chave para o desenvolvimento de aplicações imersivas e interativas para o aprendizado". Ciências \& Cognição, v. 07, i. 1, p. 105-110, 2006.

ASSIS, S. A. C. N. et al. "Efeitos do treino com jogos de videogame na cognição de idosos: revisão sistemática". Sci Med, v. 25, n. 3, 2015.

BLESS, J. J. et al. "Self-supervised, mobile-application based cognitive training of auditory attention: A behavioral and fMRI evaluation". Internet Interventions, v. 1, n. 3, p. 102-110, 2014.

BUIZA, C. et al. "Efficacy of Cognitive Training Experiences in the Elderly: Can Technology Help?", Human-Computer Interaction - INTERACT, v. 5614, p. 324333, 2014.

CARON, J.; BIDUSKI, D.; DE MARCHI, A. C. B. "Alz Memory - um aplicativo móvel para treino de memória em pacientes com Alzheimer". Revista Eletrônica de Comunicação, Informação \& Inovação em Saúde, [S.1.], v. 9, n. 2, 2015.

CASTRO, A. A. "Revisão Sistemática e Meta-análise", 2001. Disponível: http://metodolo gia.org/wp-content/uploads/2010/08/meta1.PDF, maio de 2016.

CLAREBOUT, G. et al. "The Use of iPods in Education: A Cognitive Perspective", ICLS'08 Proceedings of the 8th international conference on International conference for the learning sciences, v. 1, p. 153-158, 2015.

IRIGARAY, T. Q.; GOMES FILHO, I.; SCHNEIDER, R. H. "Efeitos de um Treino de Atenção, Memória e Funções Executivas na Cognição de Idosos Saudáveis". Psicologia: Reflexão e Crítica, v. 25, n. 1, p. 188-202, 2012.

MARTEL, M. R. F.; COLUSSI, E. L.; DE MARCHI, A. C. B. "Efeitos da intervenção com game na atenção e na independência funcional em idosos após acidente vascular encefálico". Fisioter. Pesqui., São Paulo, v. 23, n. 1, p. 52-58, 2016.

OGOMORI, K. et al. "Requirements for a Cognitive Training Game for Elderly or Disabled People", International Conference on Biometrics and Kansei Engineering, p. 150-154, 2011.

PASCUAL, M. F. et al. "Diagnosis of the attention deficit disorder using 'D2' and 'Symbols Search' tests through a game-based tool", 17th International Conference on Computer Games (CGAMES), p. 116-119, 2012.

POSSA, M. A. et al. "Comorbidades do transtorno de déficit de atenção e hiperatividade em crianças escolares", Arq Neuropsiquiatr, v. 63, i. 2, p. 479-483, 2005.

SILVA, H. Q. M. "Princípios gerais de desenvolvimento e avaliação de usabilidade de interfaces computacionais: um estudo de caso do Sistema Educacenso", 2015. Disponível: http://dspace.bc.uepb.edu.br/jspui/handle/123456789/9506, acesso: junho de 2016. 
TORRES, A. "Cognitive effects of video games on older people". International Conference on Disability, Virtual Reality \& Associated Technologies [Abstract], v. 19, p. 191-198, 2008.

WITTLAND, J. et al. "Serious Games for Cognitive Training in Ambient Assisted Living Environments - A Technology Acceptance Perspective", Human-Computer Interaction - INTERACT, v. 9296, p. 453-471, 2015.

YUE, W. S.; ZIN, N. A. M. "Voice Recognition and Visualization Mobile Apps Game for Training and Teaching Hearing Handicaps Children", Procedia Technology, v. 11, p. 479-486, 2013. 